\title{
High Accuracy Analysis of Nonconforming Mixed Finite Element Method for the Nonlinear Sivashinsky Equation
}

\author{
Lele Wang and Xin Liao \\ School of Mathematics, Zhengzhou University of Aeronautics, Zhengzhou 450046, China \\ Correspondence should be addressed to Xin Liao; woshiliaoxin@126.com
}

Received 15 February 2020; Accepted 15 April 2020; Published 30 April 2020

Academic Editor: Jan Vorel

Copyright (C) 2020 Lele Wang and Xin Liao. This is an open access article distributed under the Creative Commons Attribution License, which permits unrestricted use, distribution, and reproduction in any medium, provided the original work is properly cited.

\begin{abstract}
The fourth-order nonlinear Sivashinsky equation is often used to simulate a planar solid-liquid interface for a binary alloy. In this paper, we study the high accuracy analysis of the nonconforming mixed finite element method (MFEM for short) for this equation. Firstly, by use of the special property of the nonconforming $E Q_{1}^{\text {rot }}$ element (see Lemma 1), the superclose estimates of order $O\left(h^{2}+\Delta t\right)$ in the broken $H^{1}$-norm for the original variable $u$ and intermediate variable $p$ are deduced for the back-Euler (BE for short) fully-discrete scheme. Secondly, the global superconvergence results of order $O\left(h^{2}+\Delta t\right)$ for the two variables are derived through interpolation postprocessing technique. Finally, a numerical example is provided to illustrate validity and efficiency of our theoretical analysis and method.
\end{abstract}

\section{Introduction}

Under certain conditions, the dilute binary alloy will solidify, at which point the solid-liquid interface is unstable and has a cellular structure. When the solute rejection coefficient is close to unity, near the stability threshold, the characteristic cell size may significantly be beyond the diffusion width of the solidification zone. The Sivashinsky equation describes the dynamic of the onset and stabilization of the cellular structure, which is considered as the following fourth-order nonlinear equation $[1,2]$ :

$$
\begin{cases}u_{t}+\Delta^{2} u+\alpha u=\Delta f(u), & (X, t) \in \Omega \times(0, T], \\ u=\Delta u=0, & (X, t) \in \partial \Omega \times(0, T], \\ u(X, 0)=u^{0}(X), & X \in \Omega,\end{cases}
$$

where $\Omega$ is the interior of the rectangle $[0, a] \times[0, b]$, $X=(x, y), T>0, a>0, b>0, \alpha>0$ are fixed constants, $u^{0}(X)$ is a given smooth function, and $f(u)=(1 / 2) u^{2}-2 u$. Due to the nonlinearity of this equation, it is very difficult to find out the true solution. Thus, a lot of numerical simulation methods have been considered for (1), such as the finite difference method, finite element method (FEM for short), and region decomposition method. For one-dimensional case, Benammou and Omrani [3] studied the FEM and obtained the convergence analysis of the original variable $u$ in $L^{2}$-norm; Momani [4] presented a numerical scheme based on the region decomposition method; and Omrani and Reza and Kenan $[5,6]$ provided two kinds of finite difference schemes and proved the uniqueness and convergence, respectively. For two-dimensional case, Denet [7] gave the stability of the solution under the rectangular region; Rouis and Omrani [8] proposed a linearized three-level difference scheme; and Ilati and Dehghan [9] derived an error analysis by a meshless method based on radial point interpolation technique.

As it is known to all that in regard to the fourth-order problem, the conforming Galerkin finite element (FE for short) approximation space belongs to $H^{2}(\Omega)$, and FE solution in turn shall be $C^{1}$-continuous. This leads to the higher degree of piecewise polynomials, and the related computation is complicated and difficult (both triangular Bell element and rectangular Bogner-Fox-Schmit element [10] are typical examples). The MFEM is an optimal choice to overcome the above deficiencies, which transforms a fourth-order problem into 2 coupled second-order problems 
by introducing an intermediate variable; thus, the low-order elements can be used to solve. The nonconforming MFEM brings down the smoothness requirement on FE solution compared to the conforming case. Readers with more interests may refer [11-15] and the references listed. For problem (1), Omrani [1] developed the convergence analysis of the corresponding variables in the semidiscrete and fullydiscrete schemes by using conforming MFEM; however, situation involving nonconforming MFEM was not available till now.

It is also well known that the superconvergence analysis is an important approach to improve the precision of $\mathrm{FE}$ solution. More precisely, based on the so-called integral identity technique, the order of error in $H^{1}$-norm between FE approximation $u_{h}$ and the interpolation of the exact solution $I_{h} u$ is much better than that of $u$ and $I_{h} u$; this fascinating characteristic is called superclose. The global superconvergence will then be investigated by adding a simple postprocessing without changing the existing FE program. Meanwhile, superconvergence is critical in practical engineering numerical calculation and has always been a research hotspot. To find out more applications, readers may refer [12, 15-23]. As far as our knowledge is concerned, research on superconvergence for Sivashinsky equation is yet to be found.

The main purpose of this article is to develop a nonconforming MFE scheme for problem (1), and the superclose and superconvergence results of the original variable $u$ and auxiliary variable $p$ in the broken $H^{1}$-norm are obtained for the B-E fully-discrete scheme. The outline is organized as follows: in Section 2, the MFE spaces and variational formulation are introduced. In Section 3, based on the special property of the nonconforming $E Q_{1}^{\text {rot }}$ element (when $u \in H^{3}(\Omega)$, the consistency error is of order $O\left(h^{2}\right)$ which is one order higher than the interpolation error), the superclose results for the above two variables are deduced. In Section 4, the global superconvergence properties are derived with the help of interpolation postprocessing technique. In Section 5, a numerical example is given to verify the theoretical analysis. In the last section, a brief conclusion is drawn.

Throughout this article, $C$ denotes a positive constant that may take different values at different places but remains independent of the subdivision parameter $h$ and time step $\Delta t$. Meanwhile, we use the notations as in [10] for the Sobolev spaces $W^{m, p}(\Omega)$ with norm $\|\cdot\|_{m, p}$ and seminorm $|\cdot|_{m, p}$, where $m$ and $p$ are nonnegative integer numbers. Especially, for $p=2, p$ will be omitted in the above norms and seminorms. Furthermore, we define the space $L^{p}(a, b ; Y)$ with the norm $\|\Phi\|_{L^{p}(0, T ; Y)}=\int_{0}^{T}\|\Phi(\cdot, t)\|_{Y}^{p} \mathrm{~d} t \quad(1 \leq p<\infty)$ and $\|\Phi\|_{L^{\infty}(0, T ; Y)}=$ ess $\sup _{0<t<T}\|\Phi(\cdot, t)\|_{Y}(p=\infty)$.

\section{The MFE Spaces and Variational Formulation}

Let $\Omega$ be a rectangular domain with edges parallel to the coordinate axes, $T_{h}$ be a rectangular subdivision of $\Omega$ which need not satisfy the regular condition [10]. For all $K \in T_{h}$, $K=\left[x_{K}-h_{x_{K}}, x_{K}+h_{x_{K}}\right] \times\left[y_{K}-h_{y_{K}}, y_{K}+h_{y_{K}}\right]$, assume that the barycenter of $K$ by $\left(x_{K}, y_{K}\right)$, and the four vertices and four sides are $z_{i}, l_{i}=\overline{z_{i} z_{i+1}}(\bmod 4)(i=1,2,3,4)$, respectively. $h_{K}=\max \left\{h_{x_{K}}, h_{y_{K}}\right\}, h=\max _{K \in T_{h}} h_{K}$.

The nonconforming $E Q_{1}^{\text {rot }}$ element space [17-21, 24, 25] is defined by

$$
\begin{aligned}
V_{h}= & \left\{v_{h}:\left.v_{h}\right|_{K} \in \operatorname{span}\left\{1, x, y, x^{2}, y^{2}\right\},\right. \\
& \left.\forall K \in T_{h}, \int_{F}\left[v_{h}\right] \mathrm{d} s=0, F \subset \partial K\right\},
\end{aligned}
$$

where $\left[v_{h}\right]$ stands for the jump of $v_{h}$ across the boundary $F$ and $\left[v_{h}\right]=v_{h}$ if $F \subset \partial \Omega$.

Then, we denote the norm on $V_{h}$ as $\|\cdot\|_{h}=\left(\sum_{K \in T_{h}}|\cdot|_{1, K}^{2}\right)^{1 / 2}$.

The corresponding interpolation operator is defined as $I_{h}: v \in V=H_{0}^{1}(\Omega) \longrightarrow I_{h} v \in V_{h},\left.I_{h}\right|_{K}=I_{K}$, satisfying

$$
\begin{aligned}
\int_{l_{i}}\left(v-I_{K} v\right) \mathrm{d} s & =0, \\
\int_{K}\left(v-I_{K} v\right) \mathrm{d} x \mathrm{~d} y & =0, \\
i & =1,2,3,4, \forall K \in T_{h} .
\end{aligned}
$$

Let $p=f(u)-\Delta u$; then, the mixed variational formulation for $(1)$ is find $(u, p) \in V \times V$ such that

$$
\begin{cases}\left(u_{t}, v\right)+(\nabla p, \nabla v)+\alpha(u, v)=0, & \forall v \in V, \\ (p, q)-(\nabla u, \nabla q)=(f(u), q), & \forall q \in V, \\ u(X, 0)=u^{0}(X), & p(X, 0)=p^{0}(X)=f\left(u^{0}(X)\right)-\Delta u^{0}(X),\end{cases}
$$

where $(u, v)=\int_{\Omega} u v \mathrm{~d} x \mathrm{~d} y$.

\section{Superclose Analysis for the Fully-Discrete Approximation Scheme}

In this section, the superclose analysis for the B-E fullydiscrete scheme will be studied.
Let $\left\{t_{n} \mid t_{n}=n \Delta t ; n=0,1,2, \ldots, N\right\}$ be a uniform partition of $[0, T]$ with the time step $\Delta t=(T / N)$. For a given continuous function $u$ on $[0, T]$, we define that $u^{n}=u\left(X, t_{n}\right), \bar{\partial}_{t} u^{n}=u^{n}-u^{n-1} / \Delta t$.

The following lemma is introduced first which is important in the superclose analysis. 
Lemma 1 (see [18]). For all $v_{h} \in V_{h}$, we get

$$
\begin{gathered}
\left\|u-I_{h} u\right\|_{0}+h\left\|u-I_{h} u\right\|_{h} \leq C h^{2}\|u\|_{2}, \quad u \in H^{2}(\Omega), \\
\left(\nabla\left(u-I_{h} u\right), \nabla v_{h}\right)_{h}=0, \\
\left\|v_{h}\right\|_{0} \leq C\left\|v_{h}\right\|_{h}, \\
\sum_{K} \int_{\partial K} \frac{\partial u}{\partial n} v_{h} \mathrm{~d} s \leq C h^{2}\|u\|_{3}\left\|v_{h}\right\|_{h}, \quad u \in H^{3}(\Omega),
\end{gathered}
$$

where $(u, v)_{h}=\sum_{K} \int_{K} u v d x d y$.

Then, the B-E fully-discrete approximation scheme for (4) is find $\left(U_{h}^{n}, P_{h}^{n}\right) \in V_{h} \times V_{h}$ such that

$$
\begin{cases}\left(\bar{\partial}_{t} U_{h}^{n}, v_{h}\right)+\left(\nabla P_{h}^{n}, \nabla v_{h}\right)_{h}+\alpha\left(U_{h}^{n}, v_{h}\right)=0, & \forall v_{h} \in V_{h}, \\ \left(P_{h}^{n}, q_{h}\right)-\left(\nabla U_{h}^{n}, \nabla q_{h}\right)_{h}=\left(f\left(U_{h}^{n}\right), q_{h}\right), & \forall q_{h} \in V_{h}, \\ U_{h}^{0}=I_{h} u^{0}, & P_{h}^{0}=I_{h} p^{0} .\end{cases}
$$

Proof. Let $\quad u^{n}-U_{h}^{n}=\left(u^{n}-I_{h} u^{n}\right)+\left(I_{h} u^{n}-U_{h}^{n}\right):=\theta^{n}+$ $\rho^{n}, p^{n}-P_{h}^{n}=\left(p^{n}-I_{h} p^{n}\right)+\left(I_{h} p^{n}-P_{h}^{n}\right):=\xi^{n}+\eta^{n}$.

The error equations can be derived from (1), (6), and (9):

The existence and uniqueness of the solution for problem (9) can be found in [1]. $\left\|U_{h}^{n}-I_{h} u^{n}\right\|_{h}$ and $\left\|P_{h}^{n}-I_{h} p^{n}\right\|_{h}$.

Theorem 1. Let $\left(u^{n}, p^{n}\right)$ and $\left(U_{h}^{n}, P_{h}^{n}\right)$ be the solutions of (4) and (9), respectively, $u, p \in L^{\infty}\left(0, T ; H^{3}(\Omega)\right), u_{t}, p_{t} \in L^{2}(0, T$; $\left.H^{3}(\Omega)\right)$, and $u_{t t} \in L^{2}\left(0, T ; H^{2}(\Omega)\right)$; then, for $n=0,1,2, \ldots, N$, we have

$$
\left\|U_{h}^{n}-I_{h} u^{n}\right\|_{h}+\left\|P_{h}^{n}-I_{h} p^{n}\right\|_{h} \leq C\left(h^{2}+\Delta t\right) .
$$

Next focus will be placed on the superclose of

$$
\begin{aligned}
& \left(\bar{\partial}_{t} \rho^{n}, v_{h}\right)+\left(\nabla \eta^{n}, \nabla v_{h}\right)_{h}+\alpha\left(\rho^{n}, v_{h}\right)=-\left(\bar{\partial}_{t} \theta^{n}, v_{h}\right)-\alpha\left(\theta^{n}, v_{h}\right)+\sum_{K} \int_{\partial K} \frac{\partial p^{n}}{\partial n} v_{h} \mathrm{~d} s-\left(R_{1}^{n}, v_{h}\right), \\
& \left(\eta^{n}, q_{h}\right)-\left(\nabla \rho^{n}, \nabla q_{h}\right)_{h}=-\left(\xi^{n}, q_{h}\right)+\left(f\left(u^{n}\right)-f\left(U_{h}^{n}\right), q_{h}\right)-\sum_{K} \int_{\partial K} \frac{\partial u^{n}}{\partial n} q_{h} \mathrm{~d} s
\end{aligned}
$$

where $R_{1}^{n}=u_{t}^{n}-\bar{\partial}_{t} u^{n}=(1 / \Delta t) \int_{t_{n-1}}^{t_{n}}\left(\tau-t_{n-1}\right) u_{t t}(\tau) \mathrm{d} \tau$.

Firstly, taking $v_{h}=\eta^{n}$ in (11a) and $q_{h}=\bar{\partial}_{t} \rho^{n}$ in (11b) and then subtracting them, there holds

$$
\begin{aligned}
\left(\nabla \rho^{n}, \nabla \bar{\partial}_{t} \rho^{n}\right)_{h}+\left\|\eta^{n}\right\|_{h}^{2}= & -\left(\bar{\partial}_{t} \theta^{n}, \eta^{n}\right)-\alpha\left(\rho^{n}, \eta^{n}\right)-\alpha\left(\theta^{n}, \eta^{n}\right)+\sum_{K} \int_{\partial K} \frac{\partial p^{n}}{\partial n} \eta^{n} \mathrm{~d} s-\left(R_{1}^{n}, \eta^{n}\right) \\
& +\left(\xi^{n}, \bar{\partial}_{t} \rho^{n}\right)-\left(f\left(u^{n}\right)-f\left(U_{h}^{n}\right), \bar{\partial}_{t} \rho^{n}\right)+\sum_{K} \int_{\partial K} \frac{\partial u^{n}}{\partial n} \rho_{t} \mathrm{~d} s \\
= & \sum_{i=1}^{8} A_{i} .
\end{aligned}
$$

It is easy to verify that

$$
\left(\nabla \bar{\partial}_{t} \rho^{n}, \nabla \rho^{n}\right)_{h} \geq \frac{1}{2 \Delta t}\left(\left\|\rho^{n}\right\|_{h}^{2}-\left\|\rho^{n-1}\right\|_{h}^{2}\right),\left|A_{5}\right|=\left|\left(R_{1}^{n}, \eta^{n}\right)\right| \leq C \Delta t \int_{t_{n-1}}^{t_{n}}\left\|u_{t t}\right\|_{0}^{2} \mathrm{~d} \tau+\frac{1}{6}\left\|\eta^{n}\right\|_{h}^{2} .
$$

By virtue of Lemma 1, we arrive at 


$$
\begin{aligned}
&\left|A_{1}+A_{2}+A_{3}\right|=\mid\left.\mid \bar{\partial}_{t} \theta^{n}, \eta^{n}\right)+\alpha\left(\rho^{n}, \eta^{n}\right)+\alpha\left(\theta^{n}, \eta^{n}\right) \mid \\
& \leq C h^{4}\left(\frac{1}{\Delta t} \int_{t_{n-1}}^{t_{n}}\left\|u_{t}\right\|_{2}^{2} \mathrm{~d} \tau+\left\|u^{n}\right\|_{2}^{2}\right)+C\left\|\rho^{n}\right\|_{h}^{2} \\
&+\frac{1}{6}\left\|\eta^{n}\right\|_{h}^{2}, \\
&\left|A_{4}\right|=\left|\sum_{K} \int_{\partial K} \frac{\partial p^{n}}{\partial n} \eta^{n} \mathrm{~d} s\right| \leq C h^{4}\left\|p^{n}\right\|_{3}^{2}+\frac{1}{6}\left\|\eta^{n}\right\|_{h^{2}}^{2}
\end{aligned}
$$
holds

By using the derivative transfer technique and (5), there

$$
\begin{aligned}
\left|A_{6}+A_{8}\right|= & \left|\left(\xi^{n}, \bar{\partial}_{t} \rho^{n}\right)+\sum_{K} \int_{\partial K} \frac{\partial u^{n}}{\partial n} \bar{\partial}_{t} \rho^{n} d s\right| \\
= & \mid \bar{\partial}_{t}\left(\sum_{K} \int_{\partial K} \frac{\partial u^{n}}{\partial n} \rho^{n} \mathrm{~d} s\right)+\left(\xi^{n}, \bar{\partial}_{t} \rho^{n}\right) \\
& -\sum_{K} \int_{\partial K} \frac{\partial\left(\bar{\partial}_{t} u^{n}\right)}{\partial n} \rho^{n-1} \mathrm{~d} s \mid \\
\leq & C h^{4}\left(\frac{1}{\Delta t} \int_{t_{n-1}}^{t_{n}}\left\|u_{t}\right\|_{3}^{2} d \tau+\left\|p^{n}\right\|_{2}^{2}\right)+C\left\|\rho^{n-1}\right\|_{h}^{2} \\
& +\varepsilon\left\|\bar{\partial}_{t} \rho^{n}\right\|_{0}^{2}+\left|\bar{\partial}_{t}\left(\sum_{K} \int_{\partial K} \frac{\partial u^{n}}{\partial n} \rho^{n} \mathrm{~d} s\right)\right|
\end{aligned}
$$

In order to estimate $A_{7}$, the following assumption is given which will be proved later:

$$
\left\|U_{h}^{n}\right\|_{0, \infty}<M, \quad n=0,1,2, \ldots, N,
$$

where $M=1+\|u\|_{L^{\infty}\left(0, T ; L^{\infty}(\Omega)\right)}$.

Then, we have

$$
\begin{aligned}
\left|A_{7}\right| & =\left(f\left(u^{n}\right)-f\left(U_{h}^{n}\right), \bar{\partial}_{t} \rho^{n}\right) \\
& =-\left(\frac{1}{2}\left(\left(u^{n}\right)^{2}-\left(U_{h}^{n}\right)^{2}\right), \bar{\partial}_{t} \rho^{n}\right)+2\left(u^{n}-U_{h}^{n}, \bar{\partial}_{t} \rho^{n}\right) \\
& =-\left(\frac{1}{2}\left(\theta^{n}+\rho^{n}\right)\left(u^{n}+U_{h}^{n}\right), \bar{\partial}_{t} \rho^{n}\right)+2\left(\theta^{n}+\rho^{n}, \bar{\partial}_{t} \rho^{n}\right) \\
& \leq C\left\|\theta^{n}+\rho^{n}\right\|_{0}\left\|u^{n}+U_{h}^{n}\right\|_{0, \infty}\left\|\bar{\partial}_{t} \rho^{n}\right\|_{0}+C\left\|\theta^{n}+\rho^{n}\right\|_{0}\left\|\bar{\partial}_{t} \rho^{n}\right\|_{0} \\
& \leq C h^{4}\left\|u^{n}\right\|_{2}^{2}+\varepsilon\left\|\bar{\partial}_{t} \rho^{n}\right\|_{0}^{2}+C\left\|\rho^{n}\right\|_{h}^{2} .
\end{aligned}
$$

Substituting (13)-(18) into (12), we get

$$
\begin{aligned}
\frac{1}{2 \Delta t}\left(\left\|\rho^{n}\right\|_{h}^{2}-\left\|\rho^{n-1}\right\|_{h}^{2}\right)+\frac{1}{2}\left\|\eta^{n}\right\|_{h}^{2} \\
\leq C h^{4}\left(\left\|p^{n}\right\|_{3}^{2}+\left\|u^{n}\right\|_{2}^{2}+\frac{1}{\Delta t} \int_{t_{n-1}}^{t_{n}}\left\|u_{t}\right\|_{3}^{2} d \tau\right) \\
+C\left\|\rho^{n}\right\|_{h}^{2}+C\left\|\rho^{n-1}\right\|_{h}^{2}+\varepsilon\left\|\bar{\partial}_{t} \rho^{n}\right\|_{0}^{2} \\
+\left|\bar{\partial}_{t}\left(\sum_{K} \int_{\partial K} \frac{\partial u^{n}}{\partial n} \rho^{n} \mathrm{~d} s\right)\right| .
\end{aligned}
$$

Multiplying by $2 \Delta t$ and then summing up the above inequality, by applying discrete Gronwall's lemma, we can obtain

$$
\left\|\rho^{n}\right\|_{h}^{2}+2 \Delta t \sum_{i=1}^{n}\left\|\eta^{i}\right\|_{h}^{2} \leq C\left(h^{4}+(\Delta t)^{2}\right)+4 \varepsilon \Delta t \sum_{i=1}^{n}\left\|\bar{\partial}_{t} \rho^{i}\right\|_{0}^{2} .
$$

Secondly, taking the difference between two time levels $n$ and $n-1$ of (11b) reduces to

$$
\begin{aligned}
& \left(\bar{\partial}_{t} \eta^{n}, q_{h}\right)-\left(\nabla \bar{\partial}_{t} \rho^{n}, \nabla q_{h}\right)_{h}=-\left(\bar{\partial}_{t} \xi^{n}, q_{h}\right) \\
& \quad+\left(\bar{\partial}_{t}\left(f\left(u^{n}\right)-f\left(U_{h}^{n}\right)\right), q_{h}\right)-\sum_{K} \int_{\partial K} \frac{\partial\left(\bar{\partial}_{t} u^{n}\right)}{\partial n} q_{h} \mathrm{~d} s .
\end{aligned}
$$

Choosing $v_{h}=\bar{\partial}_{t} \rho^{n}$ in (11a) and $q_{h}=\eta^{n}$ in (21) and then adding them, we can get

$$
\begin{aligned}
\left\|\bar{\partial}_{t} \rho^{n}\right\|_{0}^{2}+\left(\bar{\partial}_{t} \eta^{n}, \eta^{n}\right)+\alpha\left(\bar{\partial}_{t} \rho^{n}, \rho^{n}\right) & \\
= & -\left(\bar{\partial}_{t} \theta^{n}, \bar{\partial}_{t} \rho^{n}\right)-\alpha\left(\theta^{n}, \bar{\partial}_{t} \rho^{n}\right)+\sum_{K} \int_{\partial K} \frac{\partial p^{n}}{\partial n} \bar{\partial}_{t} \rho^{n} \mathrm{~d} s \\
& -\left(R_{1}^{n}, \bar{\partial}_{t} \rho^{n}\right)-\left(\bar{\partial}_{t} \xi^{n}, \eta^{n}\right)+\left(\bar{\partial}_{t}\left(f\left(u^{n}\right)-f\left(U_{h}^{n}\right)\right), \eta^{n}\right) \\
& \quad-\sum_{K} \int_{\partial K} \frac{\partial\left(\bar{\partial}_{t} u^{n}\right)}{\partial n} \eta^{n} \mathrm{~d} s \\
= & \sum_{i=1}^{7} B_{i} .
\end{aligned}
$$

It is not difficult to verify that

$$
\begin{aligned}
\left(\bar{\partial}_{t} \eta^{n}, \eta^{n}\right) & \geq \frac{1}{2 \Delta t}\left(\left\|\eta^{n}\right\|_{0}^{2}-\left\|\eta^{n-1}\right\|_{0}^{2}\right), \\
\alpha\left(\bar{\partial}_{t} \rho^{n}, \rho^{n}\right) & \geq \frac{\alpha}{2 \Delta t}\left(\left\|\rho^{n}\right\|_{0}^{2}-\left\|\rho^{n-1}\right\|_{0}^{2}\right), \\
\left|B_{4}\right| & =\left|\left(R_{1}^{n}, \bar{\partial}_{t} \rho^{n}\right)\right| \leq C \Delta t \int_{t_{n-1}}^{t_{n}}\left\|u_{t t}\right\|_{0}^{2} \mathrm{~d} \tau+\frac{1}{6}\left\|\bar{\partial}_{t} \rho^{n}\right\|_{0}^{2} .
\end{aligned}
$$


By (5) and (17), there holds

$$
\begin{aligned}
\left|B_{1}+B_{2}+B_{5}\right| & =\left|\left(\bar{\partial}_{t} \theta^{n}, \bar{\partial}_{t} \rho^{n}\right)+\alpha\left(\theta^{n}, \bar{\partial}_{t} \rho^{n}\right)+\left(\bar{\partial}_{t} \xi^{n}, \eta^{n}\right)\right| \\
& \leq C h^{4}\left(\frac{1}{\Delta t} \int_{t_{n-1}}^{t_{n}}\left(\left\|u_{t}\right\|_{2}^{2}+\left\|p_{t}\right\|_{2}^{2}\right) \mathrm{d} \tau+\left\|u^{n}\right\|_{2}^{2}\right)+\frac{1}{6}\left\|\bar{\partial}_{t} \rho^{n}\right\|_{0}^{2}+C\left\|\eta^{n}\right\|_{0}^{2}, \\
\left|B_{6}\right| & \left.=\mid \bar{\partial}_{t}\left(f\left(u^{n}\right)-f\left(U_{h}^{n}\right)\right), \eta^{n}\right) \mid \\
& =\left(\bar{\partial}_{t} u^{n}\left(\frac{\theta^{n}+\theta^{n-1}}{2}+\frac{\rho^{n}+\rho^{n-1}}{2}\right)+\frac{U_{h}^{n}+U_{h}^{n-1}}{2}\left(\bar{\partial}_{t} \theta^{n}+\bar{\partial}_{t} \rho^{n}\right), \eta^{n}\right)-\left(2\left(\bar{\partial}_{t} \theta^{n}+\bar{\partial}_{t} \rho^{n}\right), \eta^{n}\right) \\
& \leq C h^{4}\left(\left\|u^{n}\right\|_{2}^{2}+\left\|u^{n-1}\right\|_{2}^{2}+\frac{1}{\Delta t} \int_{t_{n-1}}^{t_{n}}\left\|u_{t}\right\|_{2}^{2} \mathrm{~d} \tau\right)+\frac{1}{6}\left\|\bar{\partial}_{t} \rho^{n}\right\|_{0}^{2}+C\left\|\rho^{n}\right\|_{0}^{2}+C\left\|\rho^{n-1}\right\|_{h}^{2}+C\left\|\eta^{n}\right\|_{0}^{2} .
\end{aligned}
$$

From derivative transfer technique and (8), it can be proved that

$$
\begin{aligned}
\left|B_{3}\right| & =\left|\sum_{K} \int_{\partial K} \frac{\partial p^{n}}{\partial n} \bar{\partial}_{t} \rho^{n} \mathrm{~d} s\right| \\
& =\bar{\partial}_{t}\left(\sum_{K} \int_{\partial K} \frac{\partial p^{n}}{\partial n} \rho^{n} \mathrm{~d} s\right)-\sum_{K} \int_{\partial K} \frac{\partial\left(\bar{\partial}_{t} p^{n}\right)}{\partial n} \rho^{n-1} \mathrm{~d} s \\
& \leq \frac{C h^{4}}{\Delta t} \int_{t_{n-1}}^{t_{n}}\left\|p_{t}\right\|_{3}^{2} d \tau+C\left\|\rho^{n-1}\right\|_{h}^{2}+\bar{\partial}_{t}\left(\sum_{K} \int_{\partial K} \frac{\partial p^{n}}{\partial n} \rho^{n} \mathrm{~d} s\right)
\end{aligned}
$$

$\left|B_{7}\right|=\left|\sum_{K} \int_{\partial K} \frac{\partial\left(\bar{\partial}_{t} u^{n}\right)}{\partial n} \eta^{n} \mathrm{~d} s\right| \leq \frac{C h^{4}}{\Delta t} \int_{t_{n-1}}^{t_{n}}\left\|u_{t}\right\|_{3}^{2} d \tau+\left\|\eta^{n}\right\|_{h^{2}}^{2}$

Then, substituting (23)-(27) into (22) reduces to

$$
\begin{gathered}
\frac{1}{2}\left\|\bar{\partial}_{t} \rho^{n}\right\|_{0}^{2}+\frac{\alpha}{2 \Delta t}\left(\left\|\rho^{n}\right\|_{0}^{2}-\left\|\rho^{n-1}\right\|_{0}^{2}\right)+\frac{1}{2 \Delta t}\left(\left\|\eta^{n}\right\|_{0}^{2}-\left\|\eta^{n-1}\right\|_{0}^{2}\right) \\
\leq C h^{4}\left[\frac{1}{\Delta t} \int_{t_{n-1}}^{t_{n}}\left(\left\|u_{t}\right\|_{3}^{2}+\left\|p_{t}\right\|_{3}^{2}\right) \mathrm{d} \tau+\left\|u^{n}\right\|_{2}^{2}+\left\|u^{n-1}\right\|_{2}^{2}\right] \\
+C(\Delta t) \int_{t_{n-1}}^{t_{n}}\left\|u_{t t}\right\|_{0}^{2} \mathrm{~d} \tau+\bar{\partial}_{t}\left(\sum_{K} \int_{\partial K} \frac{\partial p^{n}}{\partial n} \rho^{n} \mathrm{~d} s\right) \\
+C\left\|\eta^{n}\right\|_{0}^{2}+C\left\|\rho^{n}\right\|_{0}^{2}+\left\|\eta^{n}\right\|_{h}^{2}+C\left\|\rho^{n-1}\right\|_{h}^{2} .
\end{gathered}
$$

Multiplying by $2 \Delta t$ and summing up the above inequality and then plugging (28) into (20), by discrete Gronwall's lemma again, choosing appropriate $\Delta t$ and $\varepsilon>0$ such that $1-C \varepsilon \Delta t>0$, by applying (8) and noting that $\rho^{0}=0, \eta^{0}=0$, we can obtain

$$
\Delta t \sum_{i=1}^{n}\left\|\bar{\partial}_{t} \rho^{i}\right\|_{0}^{2}+\left\|\rho^{n}\right\|_{h}^{2}+\Delta t \sum_{i=1}^{n}\left\|\eta^{i}\right\|_{h}^{2} \leq C\left(h^{4}+(\Delta t)^{2}\right) .
$$

At last, choosing $v_{h}=\bar{\partial}_{t} \eta^{n}$ in (11a) and $q_{h}=\bar{\partial}_{t} \rho^{n}$ in (21) and then substituting them, it yields

$$
\begin{aligned}
\left(\nabla \bar{\partial}_{t} \eta^{n}, \nabla \eta^{n}\right)_{h}+\left\|\bar{\partial}_{t} \rho^{n}\right\|_{h}^{2}= & -\left(\bar{\partial}_{t} \theta^{n}, \bar{\partial}_{t} \eta^{n}\right)-\alpha\left(\theta^{n}, \bar{\partial}_{t} \eta^{n}\right) \\
& -\alpha\left(\rho^{n}, \bar{\partial}_{t} \eta^{n}\right)+\sum_{K} \int_{\partial K} \frac{\partial p^{n}}{\partial n} \bar{\partial}_{t} \eta^{n} \mathrm{~d} s \\
& -\left(R_{1}^{n}, \bar{\partial}_{t} \eta^{n}\right)+\left(\bar{\partial}_{t} \xi^{n}, \bar{\partial}_{t} \rho^{n}\right) \\
& -\left(\bar{\partial}_{t}\left(f\left(u^{n}\right)-f\left(U_{h}^{n}\right)\right), \bar{\partial}_{t} \rho^{n}\right) \\
& +\sum_{K} \int_{\partial K} \frac{\partial\left(\bar{\partial}_{t} u^{n}\right)}{\partial n} \bar{\partial}_{t} \rho^{n} \mathrm{~d} s \\
= & \sum_{i=1}^{8} D_{i} .
\end{aligned}
$$

Similar to the estimates of (20) and (29), we have 


$$
\begin{aligned}
\left(\nabla \bar{\partial}_{t} \eta^{n}, \nabla \eta^{n}\right)_{h} & \geq \frac{1}{2 \Delta t}\left(\left\|\eta^{n}\right\|_{h}^{2}-\left\|\eta^{n-1}\right\|_{h}^{2}\right) \\
\left|D_{1}+D_{2}\right| & =\left|\left(\bar{\partial}_{t} \theta^{n}, \bar{\partial}_{t} \eta^{n}\right)+\alpha\left(\theta^{n}, \bar{\partial}_{t} \eta^{n}\right)\right| \\
& =\left|\bar{\partial}_{t}\left(\bar{\partial}_{t} \theta^{n}, \eta^{n}\right)+\alpha \bar{\partial}_{t}\left(\theta^{n}, \eta^{n}\right)-\left(\bar{\partial}_{t}\left(\bar{\partial}_{t} \theta^{n}\right), \eta^{n-1}\right)-\alpha\left(\bar{\partial}_{t} \theta^{n}, \eta^{n-1}\right)\right| \\
& \leq\left|\bar{\partial}_{t}\left[\left(\bar{\partial}_{t} \theta^{n}, \eta^{n}\right)+\alpha\left(\theta^{n}, \eta^{n}\right)\right]\right|+\frac{C h^{4}}{\Delta t}\left(\int_{t_{n-2}}^{t_{n}}\left\|u_{t t}\right\|_{2}^{2} \mathrm{~d} \tau+\int_{t_{n-1}}^{t_{n}}\left\|u_{t}\right\|_{2}^{2} \mathrm{~d} \tau\right)+C\left\|\eta^{n-1}\right\|_{0}^{2} \\
\left|D_{3}+D_{4}+D_{5}\right| & =\left|-\alpha\left(\rho^{n}, \bar{\partial}_{t} \eta^{n}\right)+\sum_{K} \int_{\partial K} \frac{\partial p^{n}}{\partial n} \bar{\partial}_{t} \eta^{n} \mathrm{~d} s-\left(R_{1}^{n}, \bar{\partial}_{t} \eta^{n}\right)\right| \\
& \leq\left|\bar{\partial}_{t}\left[-\alpha\left(\rho^{n}, \eta^{n}\right)+\sum_{K} \int_{\partial K} \frac{\partial p^{n}}{\partial n} \eta^{n} \mathrm{~d} s-\left(R_{1}^{n}, \eta^{n}\right)\right]\right|+C\left\|\bar{\partial}_{t} \rho^{n}\right\|_{0}^{2}+C\left\|\bar{\partial}_{t} R_{1}^{n}\right\|_{0}^{2}+\frac{C h^{4}}{\Delta t} \int_{t_{n-1}}^{t_{n}}\left\|p_{t}\right\|_{3}^{2} \mathrm{~d} \tau+C\left\|\eta^{n-1}\right\|_{h^{2}}^{2} \\
\left|D_{6}+D_{7}\right| & =\left|\left(\bar{\partial}_{t} \xi^{n}, \bar{\partial}_{t} \rho^{n}\right)-\left(\bar{\partial}_{t}\left(f\left(u^{n}\right)-f\left(U_{h}^{n}\right)\right), \bar{\partial}_{t} \rho^{n}\right)\right| \\
& \leq C h^{4}\left(\frac{1}{\Delta t} \int_{t_{n-1}}^{t_{n}}\left(\left\|p_{t}\right\|_{2}^{2}+\left\|u_{t}\right\|_{2}^{2}\right) \mathrm{d} \tau+\left\|u^{n}\right\|_{2}^{2}+\left\|u^{n-1}\right\|_{2}^{2}\right)+C\left\|\bar{\partial}_{t} \rho^{n}\right\|_{0}^{2}+C\left\|\rho^{n}\right\|_{0}^{2}+C\left\|\rho^{n-1}\right\|_{0}^{2} \\
\left|D_{8}\right| & =\left|\sum_{K} \int_{\partial K} \frac{\partial\left(\bar{\partial}_{t} u^{n}\right)}{\partial n} \bar{\partial}_{t} \rho^{n} \mathrm{~d} s\right| \leq \frac{C h^{4}}{\Delta t} \int_{t_{n-1}}^{t_{n}}\left\|u_{t}\right\|_{3}^{2} \mathrm{~d} \tau+\frac{1}{2}\left\|\bar{\partial}_{t} \rho^{n}\right\|_{h}^{2} .
\end{aligned}
$$

Substituting (31)-(35) into (30), we have

$$
\begin{aligned}
\frac{1}{2 \Delta t}\left(\left\|\eta^{n}\right\|_{h}^{2}-\left\|\eta^{n-1}\right\|_{h}^{2}\right) \leq & C h^{4}\left(\frac{1}{\Delta t} \int_{t_{n-1}}^{t_{n}}\left(\left\|p_{t}\right\|_{3}^{2}+\left\|u_{t}\right\|_{3}^{2}+\left\|u_{t t}\right\|_{2}^{2}\right) \mathrm{d} \tau+\left\|u^{n}\right\|_{2}^{2}+\left\|u^{n-1}\right\|_{2}^{2}\right) \\
& +\left|\bar{\partial}_{t}\left[\left(\bar{\partial}_{t} \theta^{n}, \eta^{n}\right)+\alpha\left(\theta^{n}, \eta^{n}\right)-\alpha\left(\rho^{n}, \eta^{n}\right)+\sum_{K} \int_{\partial K} \frac{\partial p^{n}}{\partial n} \eta^{n} \mathrm{~d} s-\left(R_{1}^{n}, \eta^{n}\right)\right]\right| \\
& +C\left\|\bar{\partial}_{t} \rho^{n}\right\|_{0}^{2}+C\left\|\rho^{n}\right\|_{0}^{2}+C\left\|\rho^{n-1}\right\|_{0}^{2} .
\end{aligned}
$$

Multiplying by $2 \Delta t$ and summing up the above inequality, by discrete Gronwall's lemma and (29), there holds

$$
\left\|\eta^{n}\right\|_{h}^{2}+\Delta t \sum_{i=1}^{n}\left\|\bar{\partial}_{t} \rho^{i}\right\|_{h}^{2} \leq C\left(h^{4}+(\Delta t)^{2}\right) .
$$

With (29) and (37), the proof is completed.

Finally, we use mathematical induction to verify assumption (15) which is similar to the technique used in $[22,23,26]$.

Let $\mu^{n}:=u^{n}-U_{h}^{n}$. Initially, when $n=0$, we have $\left\|\mu^{0}\right\|_{0, \infty}=\left\|u^{0}-I_{h} u^{0}\right\|_{0, \infty} \leq C h<1$, and the assumption is true.
Furthermore, we assume that when $n=k-1$, there holds $\left\|\mu^{k-1}\right\|_{0, \infty}<1$. Then, by Theorem 1 , we have $\left\|U_{h}^{k-1}-I_{h} u^{k-1}\right\|_{h} \leq C\left(h^{2}+\Delta t\right)$.

Additionally, we consider the situation at $n=k$. We know that $\|\mu(t)\|_{0, \infty}$ is continuous function about time $t$, so there exists $\delta>0$, for $\forall \epsilon>0$; when $\left|t_{k-1}-t_{k}\right|=\Delta t<\delta$, there holds

$$
\left|\left\|\mu^{k-1}\right\|_{0, \infty}-\left\|\mu^{k}\right\|_{0, \infty}\right|<\epsilon .
$$

Taking $\epsilon=\Delta t$ in (38), we have 


$$
\begin{aligned}
\left\|\mu^{k}\right\|_{0, \infty} \leq & \left\|\mu^{k-1}\right\|_{0, \infty}+\Delta t \leq\left\|U_{h}^{k-1}-I_{h} u^{k-1}\right\|_{0, \infty} \\
& +\left\|u^{k-1}-I_{h} u^{k-1}\right\|_{0, \infty}+\Delta t \\
\leq & C h^{-1}\left\|U_{h}^{k-1}-I_{h} u^{k-1}\right\|_{0}+C h\left|u^{k-1}\right|_{1, \infty}+\Delta t \\
\leq & C\left(h^{-1} \Delta t+h\right)+C h+\Delta t<1 .
\end{aligned}
$$

In the last step of (39), we need the time step $\Delta t$ and space step $h$ satisfy the condition $\Delta t=O\left(h^{1+\alpha}\right)(0<\alpha \leq 1)$. Above all, choose appropriate $h_{1}$ to make that $C h_{1}^{\alpha}<1$, which ends the proof.

\section{Superconvergence Analysis for the Fully- Discrete Approximation Scheme}

To obtain the superconvergence results, we combine the adjacent four elements $K_{1}, K_{2}, K_{3}$, and $K_{4}$ into a big element $\widetilde{K}$, i.e., $\widetilde{K}=\cup_{i=1}^{4} K_{i}$ (see Figure 1 ). The corresponding subdivision is defined by $T_{2 h}$.

Then, construct the interpolation postprocessing operator $I_{2 h}$ on $\widetilde{K}$ as in [27-29] which satisfies

$$
\left\{\begin{array}{l}
I_{2 h} u \mid \widetilde{K}_{2} \in P_{2}(\widetilde{K}), \\
\int_{L_{i}}\left(I_{2 h} u-u\right) \mathrm{d} s=0, \quad i=1,2,3,4, \forall \widetilde{K} \in T_{2 h} \\
\int_{K_{1} \cup K_{3}}\left(I_{2 h} u-u\right) \mathrm{d} x \mathrm{~d} y=0 \\
\int_{K_{2} \cup K_{4}}\left(I_{2 h} u-u\right) \mathrm{d} x \mathrm{~d} y=0
\end{array}\right.
$$

where $P_{2}(\widetilde{K})$ denotes the space of polynomial on $\widetilde{K}$ with degree less than or equal to 2 and $L_{i}(i=1,2,3,4)$ are the four sides of $\widetilde{K}$.

From [27], the interpolation postprocessing operator $I_{2 h}$ satisfies

$$
\begin{gathered}
I_{2 h} I_{h} u=I_{2 h} u, \\
\left\|I_{2 h} u-u\right\|_{h} \leq C h^{2}\|u\|_{3}, \\
\left\|I_{2 h} v_{h}\right\|_{h} \leq C\left\|v_{h}\right\|_{h}, \\
\forall v_{h} \in V_{h} .
\end{gathered}
$$

Theorem 2. Under the assumption of Theorem 1, there holds

$$
\left\|u^{n}-I_{2 h} U_{h}^{n}\right\|_{h}+\left\|p^{n}-I_{2 h} P_{h}^{n}\right\|_{h} \leq C\left(h^{2}+\Delta t\right) .
$$

Proof. From triangle inequality, (10), and (41), there holds

$$
\begin{aligned}
\left\|u^{n}-I_{2 h} U_{h}^{n}\right\|_{h} & =\left\|u^{n}-I_{2 h} I_{h} u^{n}+I_{2 h} I_{h} u^{n}-I_{2 h} U_{h}^{n}\right\|_{h} \\
& \leq\left\|u^{n}-I_{2 h} u^{n}\right\|_{h}+C\left\|I_{h} u^{n}-U_{h}^{n}\right\|_{h} \leq C\left(h^{2}+\Delta t\right) .
\end{aligned}
$$

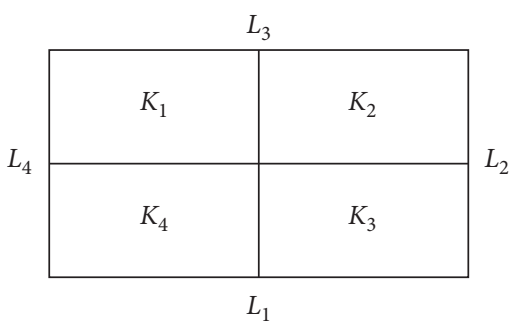

Figure 1: The big element $\widetilde{K}$.

The superconvergence result of $p$ can be obtained similarly, which completes the proof.

Remark 1.

(i) From the analysis of Theorems 1 and 2, the superclose and superconvergence results for the two variables $u$ and $p$ in the broken $H^{1}$-norm are derived, which are one order higher than the convergence results in [1].

(ii) The conclusions of this paper are applicable to other nonconforming elements such as the $Q_{1}^{\text {rot }}$ element [30], rectangular constrained $Q_{1}^{\text {rot }}$ element [31], quasi-Wilson element [32, 33], modified quasiWilson element [34], and quasi-Carey element [35].

(iii) For nonconforming linear triangle Crouzeix-Raviart element [36], Carey element [37], and Wilson element [38], the consistency errors are only of order $O(h)$; for nonconforming $P_{1}$ element [39] and $p_{1}^{\text {mod }}$ element [40], though the consistency errors reach to $\mathrm{O}\left(h^{3}\right)$ order, the interpolation error term can be estimated as $\left(\nabla\left(u-I_{h} u\right)\right.$, $\left.\nabla v_{h}\right)_{h} \leq C h|u|_{2}\left\|v_{h}\right\|_{h}$. Therefore, the superconvergence results are unable to get.

\section{Numerical Example}

Numerical simulation results are presented in this section. The Newton iterative algorithm is used to solve the nonlinear system.

Consider the following problem [1]:

$$
\begin{cases}u_{t}+\Delta^{2} u+\alpha u=\Delta f(u)+g(X), & (X, t) \in \Omega \times[0, T], \\ u(X, 0)=u^{0}(X), & X \in \Omega,\end{cases}
$$

where $\Omega=[0,1] \times[0,1], \alpha=0.5, T=1$.

Let $p=f(u)-\Delta u$; then, the mixed variational formulation for (44) is find $(u, p) \in V \times V$ such that 


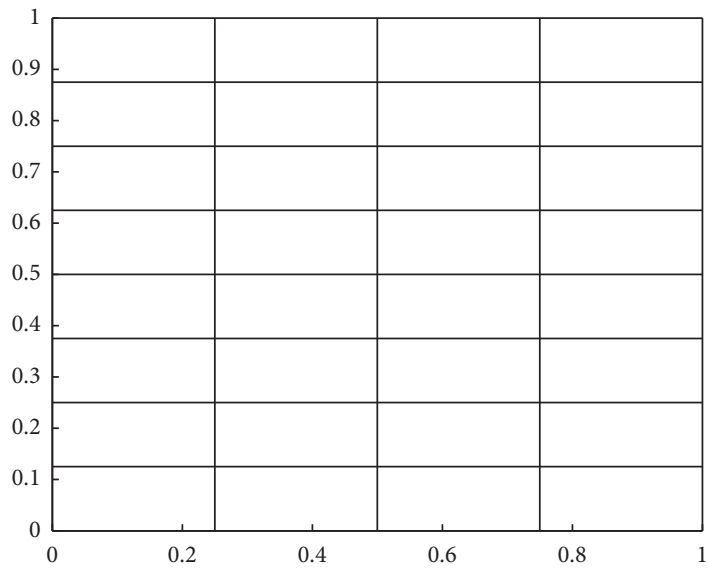

Figure 2: The rectangular mesh.

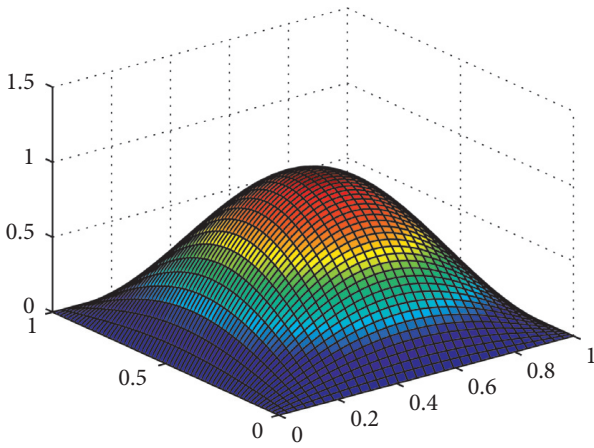

(a)

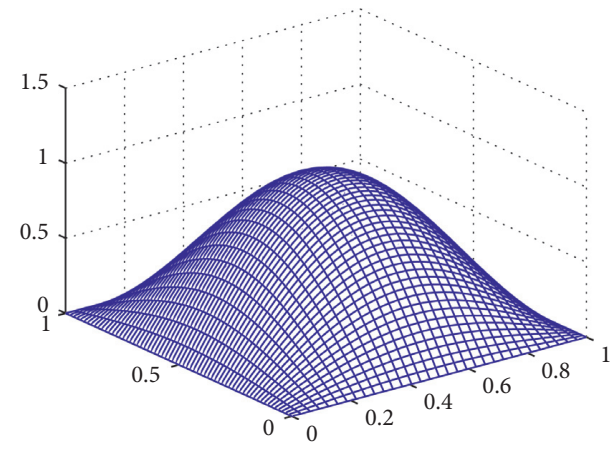

(b)

Figure 3: Exact solution $u$ (a) and numerical solution $U_{h}$ (b) on $16 \times 32$ meshes at $t=0.5$.

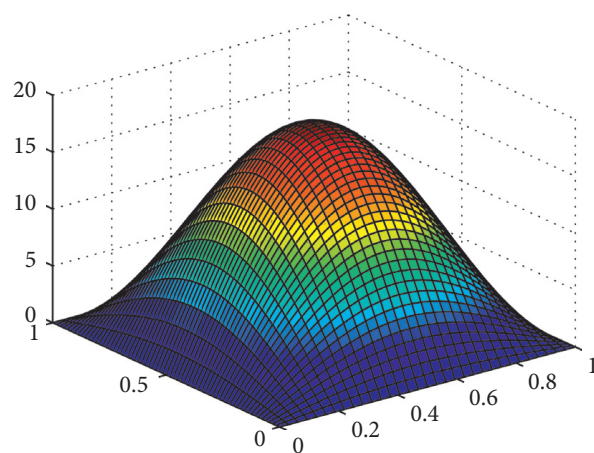

(a)

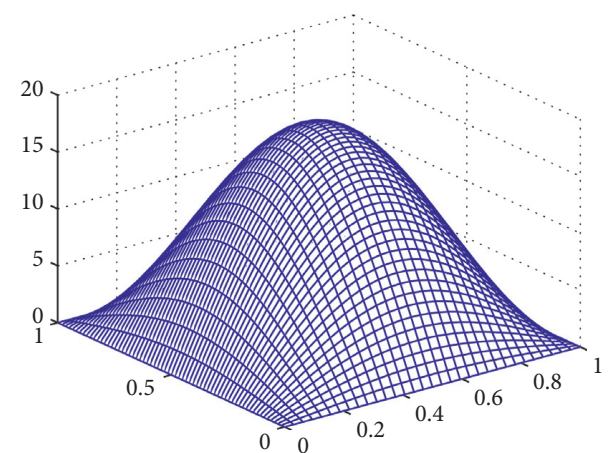

(b)

Figure 4: Exact solution $p$ (a) and numerical solution $P_{h}$ (b) on $16 \times 32$ meshes at $t=0.5$.

$$
\begin{cases}\left(u_{t}, v\right)+(\nabla p, \nabla v)+\alpha(u, v)=(g(X), v), & \forall v \in V, \\ (p, q)-(\nabla u, \nabla q)=(f(u), q), & \forall q \in V, \\ u(X, 0)=u^{0}(X), & p(X, 0)=p^{0}(X)=f\left(u^{0}(X)\right)-\Delta u^{0}(X) .\end{cases}
$$


Table 1: Numerical results of $u$ at $t=0.1$.

\begin{tabular}{lccccc}
\hline$m \times n$ & $\left\|u^{n}-U_{h}^{n}\right\|_{h}$ & Order & $\left\|U_{h}^{n}-I_{h} u^{n}\right\|_{h}$ & Order & $\left\|u^{n}-I_{2 h} U_{h}^{n}\right\|_{h}$ \\
\hline $4 \times 8$ & $4.2013 e-01$ & - & $1.4228 e-01$ & - & $2.6226 e-01$ \\
$8 \times 16$ & $2.0201 e-01$ & 1.0564 & $3.6301 e-02$ & 1.9707 & $6.7246 e-02$ \\
$16 \times 32$ & $9.9915 e-02$ & 1.0156 & $9.1169 e-03$ & 1.9933 & $1.6911 e-02$ \\
$32 \times 64$ & $4.9818 e-02$ & 1.0040 & $2.2818 e-03$ & 1.9983 & $4.2340 e-03$ \\
\hline
\end{tabular}

TABLe 2: Numerical results of $u$ at $t=0.5$.

\begin{tabular}{lccccc}
\hline$m \times n$ & $\left\|u^{n}-U_{h}^{n}\right\|_{h}$ & Order & $\left\|U_{h}^{n}-I_{h} u^{n}\right\|_{h}$ & Order & $\left\|u^{n}-I_{2 h} U_{h}^{n}\right\|_{h}$ \\
\hline $4 \times 8$ & $4.2408 e-01$ & - & $1.3655 e-01$ & - & $2.6207 e-01$ \\
$8 \times 16$ & $2.0484 e-01$ & 1.0497 & $3.5024 e-02$ & 1.9630 & $6.7315 e-02$ \\
$16 \times 32$ & $1.0147 e-01$ & 1.0134 & $9.2067 e-03$ & 1.9275 & $1.7147 e-02$ \\
$32 \times 64$ & $5.0596 e-02$ & 1.0039 & $2.3042 e-03$ & 1.9983 & $4.2931 e-03$ \\
\hline
\end{tabular}

TABLe 3: Numerical results of $p$ at $t=0.1$.

\begin{tabular}{|c|c|c|c|c|c|c|}
\hline$m \times n$ & $\left\|p^{n}-P_{h}^{n}\right\|_{h}$ & Order & $\left\|P_{h}^{n}-I_{h} p^{n}\right\|_{h}$ & Order & $\left\|p^{n}-I_{2 h} P_{h}^{n}\right\|_{h}$ & Order \\
\hline $4 \times 8$ & 7.2813 & - & 1.2981 & - & 4.1865 & - \\
\hline $8 \times 16$ & 3.6128 & 1.0110 & $3.2303 e-01$ & 2.0066 & 1.0770 & 1.9586 \\
\hline $16 \times 32$ & 1.8031 & 1.0026 & $8.0602 e-02$ & 2.0027 & $2.7098 e-01$ & 1.9908 \\
\hline $32 \times 64$ & $9.0114 e-01$ & 1.0006 & $2.0140 e-02$ & 2.0007 & $6.7852 e-02$ & 1.9977 \\
\hline
\end{tabular}

TABLe 4: Numerical results of $p$ at $t=0.5$.

\begin{tabular}{|c|c|c|c|c|c|c|}
\hline$m \times n$ & $\left\|p^{n}-P_{h}^{n}\right\|_{h}$ & Order & $\left\|P_{h}^{n}-I_{h} p^{n}\right\|_{h}$ & Order & $\left\|p^{n}-I_{2 h} P_{h}^{n}\right\|_{h}$ & Order \\
\hline $4 \times 8$ & 7.3758 & - & 1.1901 & - & 4.2129 & - \\
\hline $8 \times 16$ & 3.66791 & 1.0078 & $2.9887 e-01$ & 1.9935 & 1.0854 & 1.9565 \\
\hline $16 \times 32$ & 1.8318 & 1.0016 & $8.2041 e-02$ & 1.8651 & $2.7536 e-01$ & 1.9788 \\
\hline $32 \times 64$ & $9.1551 e-01$ & 1.0006 & $2.0500 e-02$ & 2.0006 & $6.8948 e-02$ & 1.9977 \\
\hline
\end{tabular}

The exact solutions are $u(x, y, t)=\left(1+t^{6}\right) \sin \pi x \sin \pi y$ and $p(x, y, t)=(1 / 2)\left(1+t^{6}\right)^{2}(\sin \pi x)^{2}(\sin \pi y)^{2}+2\left(\pi^{2}-1\right)$ $\left(1+t^{6}\right) \sin \pi x \sin \pi y$.
The fully-discrete approximation scheme for (45) is find $\left(U_{h}^{n}, P_{h}^{n}\right) \in V_{h} \times V_{h}$ such that

$$
\begin{cases}\left(\bar{\partial}_{t} U_{h}^{n}, v_{h}\right)+\left(\nabla P_{h}^{n}, \nabla v_{h}\right)_{h}+\alpha\left(U_{h}^{n}, v_{h}\right)=\left(g(X), v_{h}\right), & \forall v_{h} \in V_{h} \\ \left(P_{h}^{n}, q_{h}\right)-\left(\nabla U_{h}^{n}, \nabla q_{h}\right)_{h}=\left(f\left(U_{h}^{n}\right), q_{h}\right), & \forall q_{h} \in V_{h} \\ U_{h}^{0}=I_{h} u^{0}, & P_{h}^{0}=I_{h} p^{0}\end{cases}
$$

We divide the domain $\Omega$ into $m \times n$ rectangular meshes (see Figure 2). Then, the FE solutions $U_{h}^{n}, P_{h}^{n}$ can be calculated according to (46); here, we choose $\Delta t=\sqrt{\left(1 / m^{2}\right)+\left(1 / n^{2}\right)}$.

For simplicity and concreteness, we just plot the exact solutions $u, p$ and the numerical solutions $U_{h}, P_{h}$ on $16 \times 32$ meshes at $t=0.5$ (see Figures 3 and 4), respectively.
Then, the convergence, superclose, and superconvergence results of $u$ and $p$ in the broken $H^{1}$-norm at time $t=0.1$ and 0.5 are listed in Tables $1-4$, respectively.

From Tables 1 and 2, we can see that $\left\|u^{n}-U_{h}^{n}\right\|_{h}$ are convergent at order $O(h)$ and $\left\|U_{h}^{n}-I_{h} u^{n}\right\|_{h}$ and $\left\|u^{n}-I_{2 h} U_{h}^{n}\right\|_{h}$ are convergent at order $O\left(h^{2}\right)$, which coincide with the theoretical analysis. Meanwhile, the results of $\left\|u^{n}-I_{2 h} U_{h}^{n}\right\|_{h}$ are 
better than $\left\|u^{n}-U_{h}^{n}\right\|_{h}$, which indicate the superiority of the superconvergence algorithm. The results of $p$ in Tables 3 and 4 are consistent with those of $u$ in Tables 1 and 2 .

\section{Conclusions}

In this work, we study the nonconforming MFEM for fourth-order nonlinear Sivashinsky equation. The superconvergence results of the relevant variables in the broken $H^{1}$-norm are obtained, which are one order higher than those of convergence. Furthermore, a numerical example demonstrates the efficiency of the theoretical analysis.

\section{Data Availability}

The data used to support the findings of this study are available from the corresponding author upon request.

\section{Conflicts of Interest}

The authors declare no conflicts of interest regarding the publication of this paper.

\section{Acknowledgments}

This research was supported by the National Natural Science Foundation of China (Grant no. 11671369) and support program for Key Scientific Research Project of Universities in Henan Province (18A110033).

\section{References}

[1] K. Omrani, "Numerical methods and error analysis for the nonlinear Sivashinsky equation," Applied Mathematics and Computation, vol. 189, no. 1, pp. 949-962, 2007.

[2] G. I. Sivashinsky, "On cellular instability in the solidification of a dilute binary alloy," Physica D: Nonlinear Phenomena, vol. 8, no. 1-2, pp. 243-248, 1983.

[3] S. Benammou and K. Omrani, "A finite element method for the Sivashinsky equation," Journal of Computational and Applied Mathematics, vol. 142, no. 2, pp. 419-431, 2002.

[4] S. Momani, "A numerical scheme for the solution of Sivashinsky equation," Applied Mathematics and Computation, vol. 168, no. 2, pp. 1273-1280, 2005.

[5] K. Omrani, "A second-order splitting method for a finite difference scheme for the Sivashinsky equation," Applied Mathematics Letters, vol. 16, no. 3, pp. 441-445, 2003.

[6] A. Reza and Y. Kenan, "Numerical study of Sivashinsky equation using a splitting scheme based on Crank-Nicolson method," Mathematical Methods in the Applied Sciences, vol. 42, no. 16, pp. 5509-5521, 2019.

[7] B. Denet, "Sivashinsky equation in a rectangular domain," Physical Review E, vol. 75, no. 4, Article ID 046310, 2007.

[8] M. Rouis and K. Omrani, "On the numerical solution of two dimensional model of an alloy solidification problem," Modeling and Numerical Simulation of Material Science, vol. 6, no. 1, pp. 1-9, 2016.

[9] M. Ilati and M. Dehghan, "Error analysis of a meshless weak form method based on radial point interpolation technique for Sivashinsky equation arising in the alloy solidification problem," Journal of Computational and Applied Mathematics, vol. 327, pp. 314-324, 2018.
[10] P. G. Ciarlet, The Finite Element Method for Elliptic Problem, North Holland, Amsterdam, Netherlands, 1978.

[11] A. E. Diegel, C. Wang, and S. M. Wise, "Stability and convergence of a second-order mixed finite element method for the Cahn-Hilliard equation," IMA Journal of Numerical Analysis, vol. 36, no. 4, pp. 1867-1897, 2016.

[12] D. Shi, L. Wang, and X. Liao, "New estimates of mixed finite element method for fourth-order wave equation," Mathematical Methods in the Applied Sciences, vol. 40, no. 12, pp. 4448-4461, 2017.

[13] G. Chen, W. Qiu, and L. Xu, "Analysis of a mixed finite element method for the quad-curl problem," 2018, http://arxiv. org/abs/1811.06724.

[14] L. J. T. Doss and A. P. Nandini, "A fourth-order $H^{1}$-Galerkin mixed finite element method for Kuramoto-Sivashinsky equation," Numerical Methods for Partial Differential Equations, vol. 35, no. 2, pp. 445-477, 2019.

[15] D. Shi and X. Jia, "Superconvergence analysis of the mixed finite element method for the Rosenau equation," Journal of Mathematical Analysis and Applications, vol. 481, no. 1, p. 123485, 2020.

[16] N. N. Yan, Superconvergence Analysis and a Posteriori Error Estimation in Finite Element Methods, Science Press, Beijing, China, 2008.

[17] Q. Lin, L. Tobiska, and A. H. Zhou, "Superconvergence and extrapolation of non-conforming low order finite elements applied to the Poisson equation," IMA Journal of Numerical Analysis, vol. 25, no. 1, pp. 160-181, 2005.

[18] D. Y. Shi, S. P. Mao, and S. C. Chen, "An anisotropic nonconforming finite element with some superconvergence results," Journal of Computational Mathematics, vol. 23, no. 3, pp. 261-274, 2005.

[19] D. Shi, L. Wang, and X. Liao, "A new nonconforming mixed finite element scheme for second order eigenvalue problem," Applied Mathematics and Computation, vol. 273, pp. 842-855, 2016.

[20] D. Shi, J. Wang, and F. Yan, "Unconditional superconvergence analysis for nonlinear parabolic equation with $E Q_{1}{ }^{\text {rot }}$ nonconforming finite element," Journal of Scientific Computing, vol. 70, no. 1, pp. 85-111, 2017.

[21] D. Shi and J. Wang, "Unconditional superconvergence analysis for nonlinear hyperbolic equation with nonconforming finite element," Applied Mathematics and Computation, vol. 305, pp. 1-16, 2017.

[22] D. Shi, X. Liao, and L. Wang, "Superconvergence analysis of conforming finite element method for nonlinear Schrödinger equation," Applied Mathematics and Computation, vol. 289, pp. 298-310, 2016.

[23] D. Shi, X. Liao, and L. Wang, “A nonconforming quadrilateral finite element approximation to nonlinear Schrödinger equation," Acta Mathematica Scientia, vol. 37, no. 3, pp. 584-592, 2017.

[24] D. Shi and C. $\mathrm{Xu}$, " $E Q_{1}{ }^{\text {rot }}$ nonconforming finite element approximation to Signorini problem," Science China Mathematics, vol. 56, no. 6, pp. 1301-1311, 2013.

[25] D. Shi, C. Xu, and J. Chen, "Anisotropic nonconforming $E Q_{1}{ }^{\text {rot }}$ quadrilateral finite element approximation to second order elliptic problems," Journal of Scientific Computing, vol. 56, no. 3, pp. 637-653, 2013.

[26] D. Shi and H. Yang, "Superconvergence analysis of nonconforming FEM for nonlinear time-dependent thermistor problem," Applied Mathematics and Computation, vol. 347, pp. 210-224, 2019. 
[27] Q. Lin and J. F. Lin, Finite Element Methods: Accuracy and Improvement, Science Press, Beijing, China, 2006.

[28] D. Shi and Q. Tang, "NonconformingH1-Galerkin mixed finite element method for strongly damped wave equations," Numerical Functional Analysis and Optimization, vol. 34, no. 12, pp. 1348-1369, 2013.

[29] Y. Zhao, F. Wang, and D. Shi, " $E Q_{1}{ }^{\text {rot }}$ nonconforming finite element method for nonlinear dual phase lagging heat conduction equations," Acta Mathematicae Applicatae Sinica, English Series, vol. 29, no. 1, pp. 201-214, 2013.

[30] R. Rannacher and S. Turek, "Simple nonconforming quadrilateral Stokes element," Numerical Methods for Partial Differential Equations, vol. 8, no. 2, pp. 97-111, 1992.

[31] J. Hu, H. Y. Man, and Z. C. Shi, "Constrained nonconforming $Q_{1}{ }^{\text {rot }}$ element for Stokes flow and planar elasticity," Journal of Computational Mathematics, vol. 27, no. 3, pp. 311-324, 2005.

[32] S. Chen and D. Shi, "Accuracy analysis for quasi-Wilson element," Acta Mathematica Scientia, vol. 20, no. 1, pp. 44-48, 2000.

[33] D. Shi, F. Wang, and Y. Zhao, "Superconvergence analysis and extrapolation of quasi-Wilson nonconforming finite element method for nonlinear Sobolev equations," Acta Mathematicae Applicatae Sinica, English Series, vol. 29, no. 2, pp. 403-414, 2013.

[34] D. Shi and L. Pei, "Nonconforming quadrilateral finite element method for a class of nonlinear sine-Gordon equations," Applied Mathematics and Computation, vol. 219, no. 17, pp. 9447-9460, 2013.

[35] D. Shi and X. Hao, "Accuracy analysis for quasi-carey element," Journal of Systems Science and Complexity, vol. 21, no. 3, pp. 456-462, 2008.

[36] T. Apel, S. Nicaise, and J. Schöberl, "Crouzeix-Raviart type finite elements on anisotropic meshes," Numerische Mathematik, vol. 89, no. 2, pp. 193-223, 2001.

[37] D. Y. Shi, S. C. Chen, and I. Hagiwara, "Convergence analysis for a nonconforming membrane element on anisotropic meshes," Journal of Computational Mathematics, vol. 23, no. 4, pp. 373-382, 2005.

[38] Z. C. Shi, "A remark on the optimal order of convergence of Wilson nonconforming element," Mathematica Numerica Sinica, vol. 8, no. 2, pp. 159-163, 1986.

[39] C. Park and D. Sheen, "P1-Nonconforming quadrilateral finite element methods for second-order elliptic problems," SIAM Journal on Numerical Analysis, vol. 41, no. 2, pp. 624-640, 2003.

[40] P. Knobloch and L. Tobiska, "The P1mod element: a new nonconforming finite element for convection-diffusion problems," SIAM Journal on Numerical Analysis, vol. 41, no. 2, pp. 436-456, 2003. 